International Mathematical Forum, 2, 2007, no. 40, 1989 - 1995

\title{
Decentralized Stabilization of Interconnected Neutral Delay Large-Scale Systems ${ }^{1}$
}

\author{
Jinjiang Yao \\ Department of Mathematics \\ Linyi Normal University \\ Linyi, Shandong, 276005, P. R. China \\ lysyyaojinjiang@yahoo.com.cn
}

\begin{abstract}
This paper is concerned with the decentralized stabilization problem for a class of interconnected neutral delay large-scale system. Based on the linear matrix inequality (LMI) design method, the problem we address is to design a decentralized state-feedback control law such that the interconnected neutral delay large-scale system can be stabilized. A simulation example is given to illustrate the effectiveness of the approach presented in this paper.
\end{abstract}

\section{Mathematics Subject Classifications: TP 13}

Keywords: Decentralized stabilization; Interconnected systems; Neutral delay

\section{Introduction}

The states changes of some systems are affected by not only delay state but also delay differential quotient, this kind of delay system is called to be neutral delay system. Many systems can be constructed by neutral differential equation, such as steam or water pipes, heater exchangers [1], lossless transmission lines and full wave equivalent circus, equivalent electric path and so on. So the neutral delay systems are concerned widely in nearly years[1-6]. The research about neutral delay systems is concentrated in stability analysis and finding the conditions of asymptotically stable delay dependence or independence. $\mathrm{Xu}[4]$ solved the control problems of linear neutral delay system, and a design method of control based on the LMI method was provided. Based on the

\footnotetext{
${ }^{1}$ This work was supported by Natural Science Foundation of China(Grant No. 10671086).
} 
conversion methods of model and the LMI method, Han[5] gave a method to verify the stability of interconnected neutral delay large-scale system.

Because of the reliability and economical of the reality of decentralized control, it has been a very active point, and there were many researches about it[7-10]. It is very meaningful to do some decentralized control researches about interconnected neutral delay large-scale system which is made of finite subsystems; there were few reports about it. The decentralized control problems of interconnected neutral delay large-scale system are mainly talked about in this paper; we design a feedback control scaling law based on the method of LMI such that the interconnected neutral delay large-scale system can be stabilized. A simulation example is given to illustrate the effectiveness of the approach presented lastly.

\section{The description of system}

In this section, we consider a interconnected neutral delay large-scale system which is made of $\mathrm{N}$ subsystem. Its subsystem equation is

$$
\begin{gathered}
\dot{x}_{i}(t)=A_{i} x(t)+\sum_{j=1}^{N} A_{h_{i j}} x\left(t-h_{i j}\right)+A_{d_{i}} \dot{x}\left(t-d_{i}\right)+B_{i} u_{i}(t), \\
x_{i}(t)=\varphi_{i}(t), t \in[-l, 0], i=1,2, \cdots, N,
\end{gathered}
$$

where $x_{i}(t) \in R^{n_{i}}$ is state vector, $u_{i}(t) \in R^{m_{i}}$ is control impute, $A_{i}, A_{h_{i j}}, A_{d_{i}}, B_{i}$ are some matrixes which have right dimensions. $A_{h_{i j}}$ is a relevance matrix between the subsystems of $j$ and $i$, scalars $h_{i j}>0$ and $d_{i}>0$ are delay constants, $l=\max \left\{h_{i j}, d_{i}\right\}, \varphi(t) \in R^{n}$ is original value vector function which is continuously differentiable.

We will design a memoryless state feedback control to every subsystem

$$
u_{i}(t)=K_{i} x_{i}(t)
$$

in order to make the closed-loop large-scale system

$$
\dot{x}_{i}(t)=\bar{A}_{i} x(t)+\sum_{j=1}^{N} A_{h_{i j}} x\left(t-h_{i j}\right)+A_{d_{i}} \dot{x}\left(t-d_{i}\right), i=1,2, \cdots, N
$$

asymptotically stable, $\bar{A}_{i}=A_{i}-B_{i} K_{i}$. The system (1) is called to be decentralized stabilization. 


\section{The design of decentralized controller}

A condition of decentralized stabilization of system (1) is given by the following theorem.

Theorem 1. Considering interconnected neutral delay large-scale system (1), if there exists a matrix $Y_{i}$ and positive definite matrix $P_{i}>0, H_{i}>0, Q_{i}>$ 0 such that linear matrix inequality group

$$
\left(\begin{array}{ll}
\sum_{i} & T_{1 i} \\
T_{1 i}^{T} & T_{2 i}
\end{array}\right) \leq 0
$$

then the system (1) is decentralized stabilizable and $K_{i}=Y_{i} P_{i}^{-1}$, where

$$
\begin{aligned}
& \sum_{i}=P_{i} A_{i}^{T}+A_{i} P_{i}+Y_{i}^{T} B_{i}^{T}+B_{i} Y_{i}+Q_{i}+N H_{i}, \\
T_{1 i}= & \left(\left(P_{i} A_{i}^{T}+Q_{i}+N H_{i}+Y_{i}^{T} B_{i}^{T}\right) A_{d_{i}}^{T} \quad P_{i} A_{h_{i 1}}^{T} \cdots P_{i} A_{h_{i n}}^{T}\right), \\
& T_{2 i}=\operatorname{diag}\left(Q_{i}-A_{d_{i}}\left(Q_{i}+N H_{i}\right) A_{d_{i}}^{T}, H_{1} \cdots H_{N}\right) .
\end{aligned}
$$

Proof Considering the condition of system of (1), let the control level to be (2), the closed-loop compound of interconnected neutral delay large-scale system (1) is (3), it is easy to know that the stability of the system (3) and the stability of

$$
\dot{x}_{i}(t)=\bar{A}_{i}^{T} x(t)+\sum_{j=1}^{N} A_{h_{i j}}^{T} x\left(t-h_{i j}\right)+A_{d_{i}}^{T} \dot{x}\left(t-d_{i}\right)
$$

are equal.

Assume $\wp_{i}\left(x_{i}(t)\right)=x_{i}(t)-A_{d_{i}}^{T} x\left(t-d_{i}\right)$, then system (5) is

$$
\dot{\wp}_{i}\left(x_{t}\right)=\bar{A}_{i}^{T} x_{i}+\sum_{j=1}^{N} A_{h_{i j}}^{T} x\left(t-h_{i j}\right), i=1,2, \cdots, N .
$$

Because of the LMI (4), we know $Q_{i}-A_{d_{i}} Q_{i} A_{d_{i}}^{T}<0$ easily. So

$$
\wp_{i}\left(x_{i}(t)\right)=x_{i}(t)-A_{d_{i}}^{T} x\left(t-d_{i}\right)
$$

is stable.

Considering the following Lyapunov-Krasovskii function

$$
\begin{aligned}
V\left(x_{t}, t\right)= & \sum_{i=1}^{N}\left[\wp^{T}\left(x_{i}(t)\right) P_{i} \wp\left(x_{i}(t)\right)\right. \\
& +\sum_{j=1}^{N} \int_{-h_{i j}}^{0} x_{j}^{T}(t+\theta) H_{i} x_{j}(t+\theta) d \theta \\
& \left.+\int_{-d_{i}}^{0} x_{i}^{T}(t+\theta) Q_{i} x_{i}(t+\theta) d \theta\right],
\end{aligned}
$$


we note $\wp_{i}\left(x_{i}(t)\right)=\wp_{i}, x_{i}\left(t-d_{i}\right)=x_{d_{i}}$ for short, then

$$
\begin{aligned}
& \dot{V}=\sum_{i=1}^{N}\left\{2 \wp^{T}\left(x_{i}(t)\right) P \dot{\rho}\left(x_{i}(t)\right)+\sum_{j=1}^{N}\left[x_{j}^{T} H_{j} x_{j}+x_{j}\left(t-h_{i j}\right)^{T} H_{j} x_{j}\left(t-h_{i j}\right)\right]\right. \\
& \left.+x_{i}^{T} Q_{i} x_{i}-x_{i}\left(t-d_{i}\right)^{T} Q_{i} x_{i}\left(t-d_{i}\right)\right\} \\
& =\sum_{i=1}^{N}\left\{2 \wp_{i}^{T} P_{i} \bar{A}_{i}^{T}\left(\wp_{i}+A_{d_{i}}^{T} x_{d_{i}}\right)+2 \sum_{j=1}^{N}\left(\wp_{i}^{T} P_{i} A_{h_{i j}}^{T} x_{j}\left(t-h_{i j}\right)\right.\right. \\
& \left.+\sum_{j=1}^{N}\left[x_{j}^{T} H_{j} x_{j}-x_{j}\left(t-h_{i j}\right)^{T} H_{j} x_{j}\left(t-h_{i j}\right)\right]+x_{i}^{T} Q_{i} x_{i}-x_{i}\left(t-d_{i}\right)^{T} Q_{i} x_{i}\left(t-d_{i}\right)\right\} \\
& =\sum_{i=1}^{N}\left\{\wp_{i}^{T}\left(P_{i} \bar{A}_{i}^{T}+\bar{A}_{i}^{T} P_{i}\right) \wp_{i}+2 \wp_{i}^{T} P_{i} \bar{A}_{i}^{T} A_{d_{i}}^{T} x_{d_{i}}+2 \sum_{j=1}^{N} \wp_{i}^{T} P_{i} A_{h_{i j}}^{T} x_{j}\left(t-h_{i j}\right)\right. \\
& +\sum_{j=1}^{N}\left[\left(\wp_{j}+A_{d_{j}}^{T} x_{d_{j}}\right)^{T} H_{j}\left(\wp_{j}+A_{d_{j}}^{T} x_{d_{j}}\right)\right]-\sum_{j=1}^{N} x_{j}^{T}\left(t-h_{i j}\right) H_{j} x_{j}\left(t-h_{i j}\right) \\
& \left.+\left[\left(\wp_{i}+A_{d_{i}}^{T} x_{d_{i}}\right)^{T} Q_{i}\left(\wp_{i}+A_{d_{i}}^{T} x_{d_{i}}\right)\right]-x_{d_{i}}^{T} Q_{i} x_{d_{i}}\right\} \\
& =\sum_{i=1}^{N}\left\{\wp_{i}^{T}\left(P_{i} \bar{A}_{i}^{T}+\bar{A}_{i} P_{i}+Q_{i}\right) \wp_{i}+\sum_{j=1}^{N} \wp_{j}^{T} H_{j} \wp_{j}+2 \wp_{i}^{T}\left(P_{i} \bar{A}_{i}^{T}+Q_{i}\right) A_{d_{i}}^{T} x_{d_{i}}\right. \\
& +2 \sum_{j=1}^{N} \wp_{j}^{T} H_{j} A_{d_{j}}^{T} x_{d_{j}}+2 \sum_{j=1}^{N} \wp_{i}^{T} P_{i} A_{h_{i j}}^{T} x_{j}\left(t-h_{i j}\right)-\sum_{j=1}^{N} x_{j}^{T}\left(t-h_{i j}\right) H_{j} x_{j}\left(t-h_{i j}\right) \\
& \left.+\sum_{j=1}^{N} x_{d_{j}}^{T} A_{d_{j}} H_{j} A_{d_{j}}^{T} x_{d_{j}}+x_{d_{i}}^{T} A_{d_{i}} Q_{i} A_{d_{i}}^{T} x_{d_{i}}-x_{d_{i}}^{T} Q_{i} x_{d_{i}}\right\} \\
& =\sum_{i=1}^{N}\left(\wp_{i}^{T}\left(P_{i} \bar{A}_{i}^{T}+\bar{A}_{i} P_{i}+Q_{i}\right) \wp_{i}+\sum_{i=1}^{N} \wp_{i}^{T} H_{i} \wp_{i}+2 \wp_{i}^{T}\left(P_{i} \bar{A}_{i}^{T}+Q_{i}\right) A_{d_{i}}^{T} x_{d_{i}}\right. \\
& +2 \sum_{j=1}^{N} \wp_{i}^{T} H_{i} A_{d_{j}}^{T} x_{d_{j}}+2 \sum_{j=1}^{N} \wp_{i}^{T} P_{i} A_{h_{i j}}^{T} x_{j}\left(t-h_{i j}\right)-\sum_{j=1}^{N} x_{j}^{T}\left(t-h_{i j}\right) H_{j} x_{j}\left(t-h_{i j}\right) \\
& -x_{d_{i}}^{T}\left(Q_{i}-A_{d_{i}}\left(Q_{i}+N H_{i}\right) A_{d_{i}}^{T}\right) x_{d_{i}} .
\end{aligned}
$$

Moreover, using $\alpha \beta+\beta^{T} \alpha^{T} \leq \alpha \alpha^{T}+\beta^{T} \beta, \alpha, \beta$ are matrix which have probable dimension, then

$$
\dot{V} \leq \sum_{i=1}^{N} \wp_{i}^{\top} \prod \wp_{i}
$$

where

$$
\begin{aligned}
\Pi= & P_{i} \bar{A}_{i}^{T}+\bar{A}_{i} P_{i}+Q_{i}+N H_{i} \\
& +\left(P_{i} \bar{A}_{i}^{T}+Q_{i}+N H_{i}\right) A_{d_{i}}^{T}\left(Q_{i}-A_{d_{i}}\left(Q_{i}+N H_{i}\right) A_{d_{i}}^{T}\right)^{-1} A_{d_{i}} \\
& \times\left(P_{i} \bar{A}_{i}^{T}+Q_{i}+N H_{i}\right)^{T}+\sum_{j=1}^{N} P_{i} A_{h_{i j}}^{T} H_{j}^{-1} A_{h_{i j}} P_{i} .
\end{aligned}
$$


If $\prod<0$, then the proof could be finished by the stability theorem of $[7, \mathrm{Th} 7.1$ ], because difference operator is stable.

By the schur complementary lemma, it is easy to know that $\prod<0$ is equal to

$$
\left(\begin{array}{cc}
\Xi_{i} & \Gamma_{1 i} \\
\Gamma_{1 i}^{T} & \Gamma_{2 i}
\end{array}\right)<0
$$

where

$$
\begin{aligned}
& \Xi_{i}=P_{i} \bar{A}_{i}^{T}+\bar{A}_{i}^{T} P_{i}+Q_{i}+\sum_{j=1}^{N} H_{i}, \\
& \Gamma_{1 i}=\left(P_{i} \bar{A}_{i}^{T}+Q_{i}+N H_{i} \quad P_{i} A_{h_{i 1}}^{T} \cdots P_{i} A_{h_{i N}}^{T}\right) \frac{n !}{r !(n-r) !}, \\
& \Gamma_{2 i}=\operatorname{diag}\left(Q_{i}-A_{d_{i}}\left(Q_{i}+N H_{i}\right) A_{d_{i}}^{T}, H_{1} \cdots H_{N}\right) .
\end{aligned}
$$

Let $K_{i} P_{i}=Y_{i}$, then $\prod<0$ from LMIs (4) and (7).

Remark. !!It is easy to transform the condition of (4) to LMI condition. In addition, the stability of difference operator can be seen in [4].

\section{Example}

Considering a interconnected neutral delay large-scale system which is made of two subsystem

$$
\dot{x}_{i}(t)=A_{i} x(t)+\sum_{j=1}^{2} A_{h_{i j}} x(t-h)+A_{d_{i}} \dot{x}(t-d)+B_{i} u(t), \quad i=1,2 ; j=1,2,
$$

where

$$
\begin{aligned}
& A_{1}=\left(\begin{array}{cc}
-1 & 1 \\
1 & -1
\end{array}\right), A_{h_{11}}=\left(\begin{array}{cc}
0.5 & 0 \\
0 & -0.2
\end{array}\right), A_{d_{1}}=\left(\begin{array}{cc}
-0.1 & 0.5 \\
0.1 & 0
\end{array}\right) \\
& B_{1}=1, A_{2}=\left(\begin{array}{cc}
-1 & 1.5 \\
1 & -1
\end{array}\right), A_{h_{21}}=\left(\begin{array}{cc}
0 & 0.2 \\
0 & -0.1
\end{array}\right), A_{h_{22}}=\left(\begin{array}{ll}
0.4 & 0 \\
0.5 & 0
\end{array}\right), \\
& A_{d_{2}}=\left(\begin{array}{cc}
0 & 0.5 \\
0.1 & 0.2
\end{array}\right), B_{2}=1 .
\end{aligned}
$$

It is to know that two subsystems are unstable, by solving the LMI (4), we have

$$
\begin{aligned}
& P_{1}=\left(\begin{array}{cc}
21.5916 & 0 \\
0 & 31.1878
\end{array}\right), P_{2}=\left(\begin{array}{cc}
21.5916 & 0 \\
0 & 31.1878
\end{array}\right), H_{1}=\left(\begin{array}{cc}
38.2569 & 0.6315 \\
0.6315 & 27.3701
\end{array}\right), \\
& H_{2}=\left(\begin{array}{cc}
39.2966 & 0 \\
0 & 39.2966
\end{array}\right), Q_{1}=\left(\begin{array}{cc}
47.4751 & 0.7761 \\
0.7761 & 34.0535
\end{array}\right), Q_{2}=\left(\begin{array}{cc}
47.4175 & 2.9265 \\
2.9265 & 34.3461
\end{array}\right) \text {, } \\
& Y_{1}=\left(\begin{array}{cc}
-60.3728 & 0 \\
0 & 31.1878
\end{array}\right), Y_{2}=\left(\begin{array}{cc}
-81.8122 & -5.4272 \\
0.7544 & -68.3873
\end{array}\right) \text {. }
\end{aligned}
$$




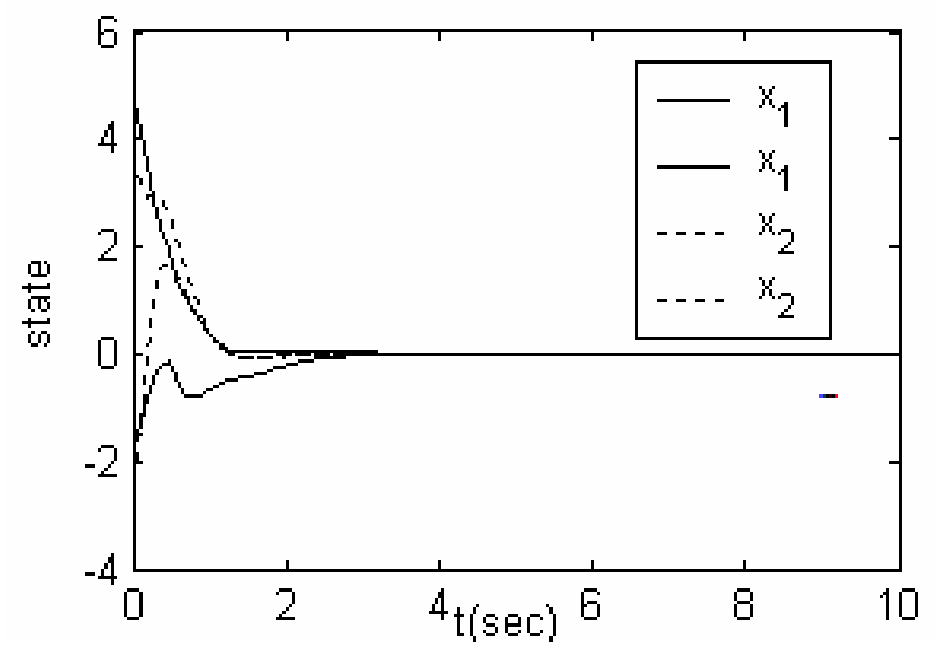

Figure 1.

Moreover

$$
K_{1}=Y_{1} P_{1}^{-1}=\left(\begin{array}{cc}
-2.7961 & 0.6312 \\
-1.5755 & -1.1423
\end{array}\right), K_{2}=Y_{2} P_{2}^{-1}=\left(\begin{array}{cc}
-3.7891 & -0.1740 \\
0.0349 & -2.1607
\end{array}\right),
$$

the decentralized state-feedback control laws are $u_{1}=k_{1} x_{1}$ and $u_{2}=k_{2} x_{2}$, choose initial condition as $x_{1}\left(t_{0}\right)=[3,2]^{T}, x_{2}\left(t_{0}\right)=[4,5]^{T}, h_{i j}=0.5, d_{i}=0.2$.

The state of two subsystems are presented in figure 1 , and the real line is the state of subsystem one, the imagine line is the state of subsystem of another. It is obviously that the system could be stabilized by the designed decentralized state-feedback control law.

\section{Conclusion}

Decentralized stabilization problem for a class of interconnected neutral delay large-scale system is considered in this paper, a condition of asymptotically stable of interconnected neutral delay large-scale system is given and the result is given in the form of LMI. It is easy to solve and could be applied easily. But we only considered a simple interconnected neutral delay large-scale system, the complicated interconnected neutral delay large-scale system will be studied in the nearly future.

\section{References}

[1] V. B. Kolmanovskii, J. P. Richard and A. Tchangani, Stability of linear systems with discrete- !! plus- distribu ted delays: Application to some 
model transformations, Proceedings of MTNS 98, Padova, Italy, (2000), 2326-2332.

[2] A. Bellen, N. Guglielmi and A. E. Ruheli, Methods for linear systems of circuits delay differential equations of neutral type, IEEE Transactions on Circuits and Systems, 46 (1999), 212-216.

[3] Z. D. Wang, J. Lam, K. J. Burnham, Stability analysis and observer design for neutral delay systems, IEEE Transactions on Automatic Control, 47(3) (2002), 478-483.

[4] S. Y. Xu , J. Lam and C. W. Yang, $H_{\infty}$ and positive-real control for linear neutral delay systems, IEEE Tr -ansactions on Automatic Control, 46 (2001), 1321-1326.

[5] Q. L. Han, Robust stability of uncertain delay-differential systems of neutral type, Automatica, 38 (2002), 719-723.

[6] I. Dan etc, On delay-dependent stability for linear neutral systems, Automatica, 39 (2003) 255- 261.

[7] J. Hale, Theory of Functional Differential Equations, Springer-Verlag, New York, 1977.

[8] B. Xu, Y. Xu and Y. Zhou Decentralized stabilization of large-scale interconnected time-delay systems: an LMI approach, Control Theory and Applications, 19 (2002).

[9] J. Yan, H. T. Jason Sheng and C. Fan, Robust stabilization of large-scale systems with nonlinear uncertainties via decentralized state feedback, J. Franklin Inst., 3358 (1998), 951-961.

[10] F. Liu , ect,Decentralized robust control for uncertain delay composite systems, Control And Decision, 16 (2001).

\section{Received: January 9, 2007}

